\title{
A Possible Experimental Way to Measure the One-Way Speed of Light
}

\author{
J. R. Croca ${ }^{1,2}$, R. N. Moreira ${ }^{2}$, M. Gatta ${ }^{2,3}$ and P. Castro ${ }^{2}$ \\ ${ }^{1}$ Department of Physics of the Faculty of Sciences of the University of Lisbon, Portugal \\ ${ }^{2}$ Center of Philosophy of Sciences of the University of Lisbon, Portugal \\ ${ }^{3}$ CINAV/Escola Naval (Portuguese Naval Academy), Almada, Portugal \\ Email: jncroca@fc.ul.pt
}

\begin{abstract}
The speed of light has been repeatedly measured considering the time a photon spends travelling from an initial point to another and then coming back to the initial point, the so called two-way speed of light. Experiments aiming to measure the speed of light when it travels strictly an initial point to another, the so called one-way speed of light, have also been proposed. Here we will discuss the conceptual basics some of these experiments, proposing what seems to be a new feasible procedure to measure the one-way speed of light. According to Special Relativity it is expected that the measurable value will match the observed value for the two-way speed of light.
\end{abstract}

Keywords: One-way speed of light, two-way speed of light, clock calibration, conceptual experiments, practical experiments, absolute space, relative space.

\section{Introduction}

One of the first known concrete experiments for determination the speed of light was done by Galileo Galilee. Since then, many experiments for studying the light speed behavior have been proposed. Michelson in his book[1] gives a good account of some of these experiments and of the ideas behind them. A very important experiment in the conceptual development of physics was done by Michelson and Morley[2]. This experiment aimed to study the effect of a hypothetical luminiferous aether on the light speed. It is well known that the experiment gave the so-called null results. That is, no aether wind effect was detected. Inspired by the results of this experiment Einstein developed the special theory of relativity[3].

\section{Proposed Experiments for Studying the Possible One-Way Speed of Light Variation}

A seemingly easy way to determinate the one-way velocity of light would eventually be the one depicted in Fig. 1, below.

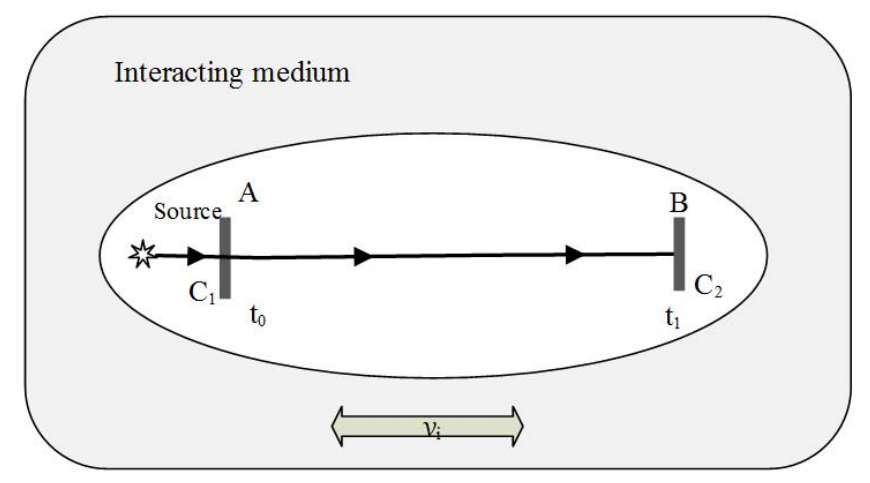

Figure 1. Eventual set up for the one-way speed of light determination 
On a platform, a source and two clocks, $\mathrm{C}_{1}$ and $\mathrm{C}_{2}$, are placed at points $\mathrm{A}$ and $\mathrm{B}$. In this case, the light photon, emitted by the source reaches the point $\mathrm{A}$, in which the first clock $\mathrm{C}_{1}$ is placed and then marks the initial time $t_{0}=0$. Then the light photon keeps moving towards the point $\mathrm{B}$. Upon arriving at clock $\mathrm{C}_{2}$, after traveling the time a distance $\mathrm{L}$, marks the time $t_{1}$, and the measured time difference is, $\Delta t=t_{1}-t_{0}$.

Nevertheless, this apparent simple measurement process is only an illusion[4], because, in reality, things are rather more complex.

To measure the initial time $t_{0}=0$, we need a clock $\mathrm{C}_{1}$ localized at point $\mathrm{A}$. To determinate the travelling time, another clock $\mathrm{C}_{2}$ must be placed at the arriving point $\mathrm{B}$. Then the total transit time would be given by the two-time readings $\Delta t=t_{1}-t_{0}$. This conclusion would only be correct only if the two clocks were synchronized. This rises the magnum problem of how to synchronize two separated clocks[5].

An eventual procedure for synchronizing the two clocks would eventually be: Synchronizing the two clocks at the same position A. Then, by slowly moving the clock B to the final position we would get them synchronized at the two different positions. Nevertheless, it has been shown that whether this displacement is done in slow motion or in fast motion there is always an indeterminate amount of time necessary to calibrate the clock B relative to clock A. Furthermore, this time, needed to synchronize the two separated clocks, depends on the theory. The traditional way to synchronize two separated clocks is given by special theory of relativity[6]. In this situation Einstein convention is accepted by assuming that the two opposite one-way speeds is the same, that is the two-way speed of light is invariant and equal to $c$ in every circumstance. Still, as shown by Reichenbach[7] and Selleri[8], there are many other open possibilities, compatible with the assumption of the two-way velocity of light invariance.

In such conditions, since the synchronization of the two separated clocks depends on the theory, it follows immediately that the one-way velocity of light, depends also on it. It was precisely because of being aware of these basic facts that, back in 1898, Henri Poincaré[9] arrived at the conclusion that it was impossible to measure the one-way velocity of light. Since then, this impossibility statement is known as Poincaré's curse.

Even if the correct calibrated time readings at clock $\mathrm{C}_{2}$ are not known because of the clock calibration problem, the eventual difference among different time readings for diverse values of the relative velocity, $v_{i}$, to the medium could, in principle, be experimentally observed. This conclusion is a consequence of the fact that in the experiment the two clocks remain always at rest relatively to each other and that furthermore, we assume that they are good clocks keeping always the same rhythm. In such a case the calibration term is constant for any measurement conditions.

Suppose now that, the experimental platform moves relatively to surrounding interacting medium, with different velocities. This could be accomplished by placing the experimental device on a train, an aircraft or other device. In such a case, the first clock $\mathrm{C}_{1}$ is activated at time, say, $t_{0}$, and afterwards clock $\mathrm{C}_{2}$ is activated indicating the time $t_{1}^{\prime}$. We know that this time is not in general the correct time. The correct time would be $t_{1}^{\prime}=t_{1} \pm \varepsilon_{0}$, where $\varepsilon_{0}$ represents the term necessary for a correct clock calibration.

For a velocity $v_{1}$ relative to the medium, the observed time difference between emission and reception of a light pulse is

$$
\Delta t_{v_{1}}=t_{1, v_{1}}^{\prime}-t_{0, v_{1}}=t_{1, v_{1}} \pm \varepsilon_{0}-t_{0, v_{1}},
$$

while for a different velocity $v_{2}$ relative to medium, could eventually be

$$
\Delta t_{v_{2}}=t_{1, v_{2}}^{\prime}-t_{0, v_{2}}=t_{1, v_{2}} \pm \varepsilon_{0}-t_{0, v_{2}} .
$$

The difference in the two readings for these two eventually diverse velocities is given by

$$
\Delta t=\Delta t_{v_{1}}-\Delta t_{v_{2}}=\left(t_{1, v_{1}} \pm \varepsilon_{0}-t_{0, v_{1}}\right)-\left(t_{1, v_{2}} \pm \varepsilon_{0}-t_{0, v_{2}}\right)
$$

or

$$
\Delta t=\left(t_{1, v_{1}}-t_{1, v_{2}}\right)-\left(t_{0, v_{1}}-t_{0, v_{2}}\right)
$$


independent of the calibration term. If in each measurement we set clock $\mathrm{C}_{1}$ at zero we shall have simply

$$
\Delta t=t_{1, v_{1}}-t_{1, v_{2}}
$$

Therefore, the eventual difference in time measurements, for diverse possible interactions with the medium, would indeed be a function of the relative velocity

$$
\Delta t=\Delta t\left(v_{i}\right) .
$$

\section{Beyond Poincaré's Curse}

An open possibility to circumvent Poincaré's curse is depicted in Fig. 2, below.

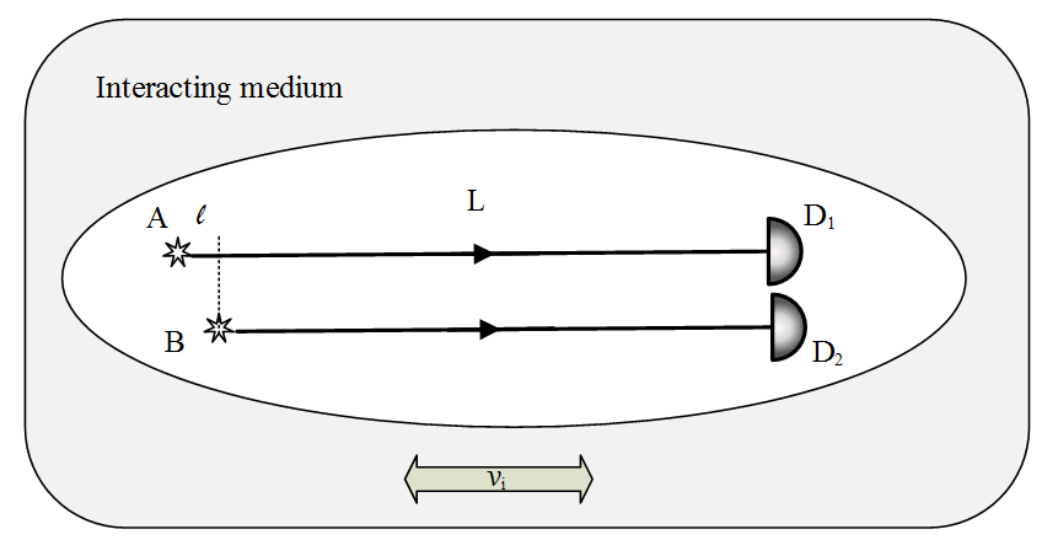

Figure 2. One-way setup

As shown in Fig. 2, the two detectors, $D_{1}$ and $D_{2}$, that stand for the clocks, are placed practically at the same position. In addition, the two points of light sources, A and B, are also placed very near, because the distance between them is $\ell$, which is relatively very small. Furthermore, these two sources emit at the same time.

A practical sketch for a possible device for doing such a task is shown in Fig. 3.

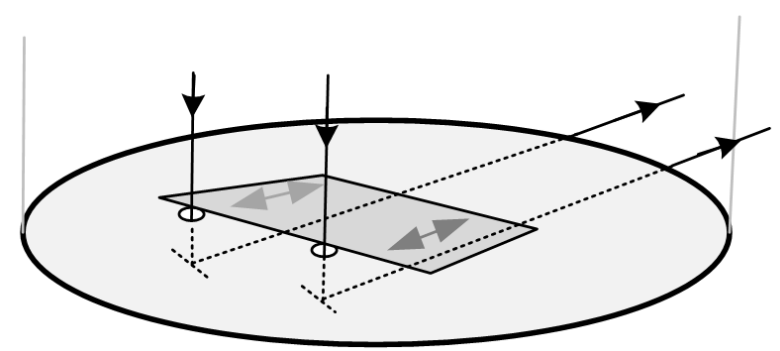

Figure 3. Practical setup for producing two light source emitting at the same time

A plane wave impinges on an opaque surface with two holes. The shutter moves opening or closing the holes. Behind the holes there are two mirrors directing the light.

Let us now estimate the travel time for the setup indicated in Fig. 2. The light from point A reaches the detector, after traveling the distance, $L+\ell$, with $L \gg \ell$, while the light emitted from point B travels the distance $L$. Since the detectors are not synchronized with A and B, they read only the times $t_{1}^{\prime}$ and $t^{\prime}{ }_{2}$. As the light is travelling only through the air these times are given by,

$$
t^{\prime}{ }_{1}=\frac{\ell+L}{c_{\rightarrow}}+\varepsilon_{0}=t_{1}+\varepsilon_{0} \text { and } t^{\prime}{ }_{2}=\frac{L}{c_{\rightarrow}}+\varepsilon_{0}=t_{2}+\varepsilon_{0}
$$

where $t_{1}$ and $t_{2}$ are the correct calibrated arrival times and $\varepsilon_{0}$ is the synchronizing term. The observed difference between the two-time readings, $\Delta t$ at each detector is

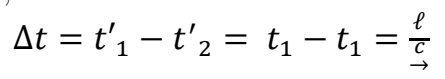


consequently, the one-way velocity of light $c_{\rightarrow}$ is given by,

$$
c_{\rightarrow}=\frac{\ell}{\Delta t}
$$

With this experimental process it is seams eventually possible to overcome Poincaré curse. In any case, just as before, a hypothetical change in the one-way velocity of light seems indeed possible to evaluate.

Let us now estimate the relative error in the time measurement with this experimental process. Since the best available photonic detects have a precision of about

$$
\Delta t_{0} \approx 10 P s=10^{-11} s
$$

and by assuming that $\ell=3 \mathrm{~cm}$ the relative time error is about, $\varrho \sim .1$. If one wishes to increase the precision of the measurement it is necessary to increase the size of $\ell$, which due to the clock calibration procedure may be not very convenient.

Another way to increase the measurement precision is to use interferometric procedures, as shown in Fig. 4, below.

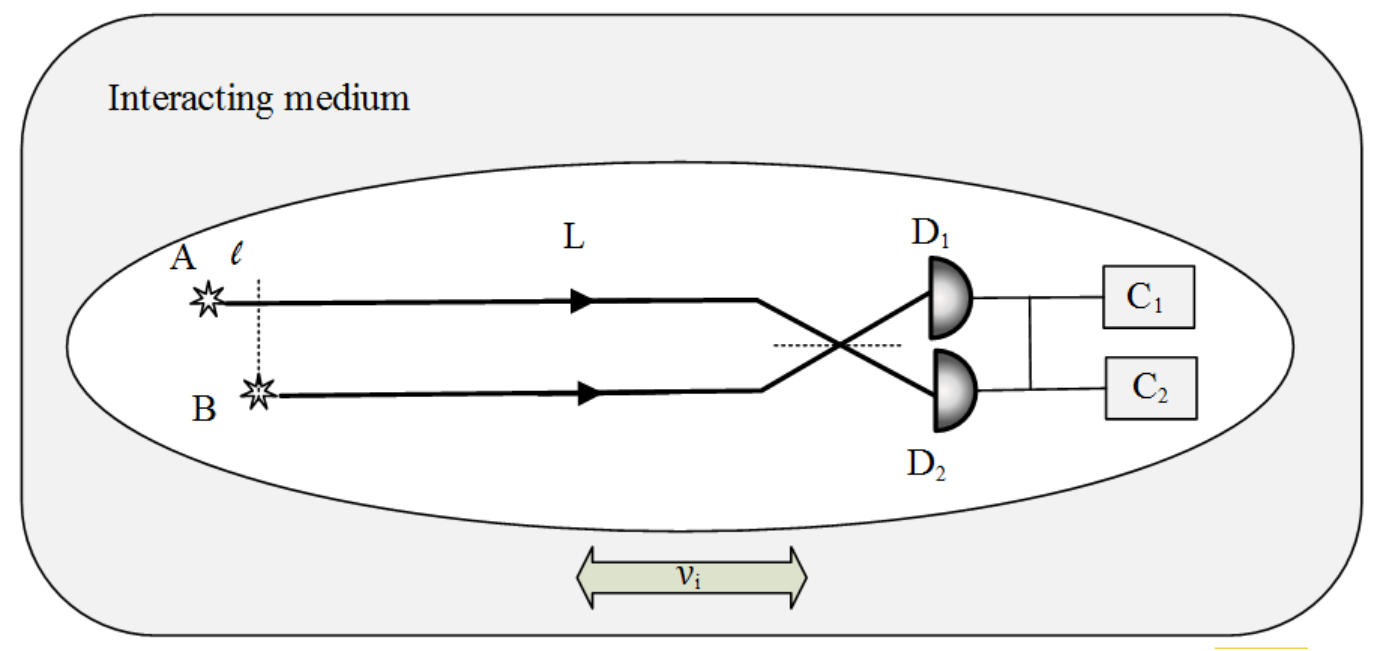

Figure 4. Interferometric setup for measuring the one-way speed of light

A steady laser beam, with coherence length much greater than $\ell$ is injected on the apparatus, so that the two-point sources A and B, emit at the same time. Since the light may be considered as practically monochromatic the intensities seen by the detectors may be represented by

$$
\left\{\begin{array}{l}
I_{1}=2 I_{0}(1+\gamma \cos \delta) \\
I_{2}=2 I_{0}(1-\gamma \cos \delta)
\end{array}\right.
$$

with standing for the $\gamma$ visibility factor. The difference between the two readings

where the phase difference $\delta$ can be written

$$
\Delta=I_{1}-I_{2}=4 I_{0} \gamma \cos \delta
$$

$$
\delta=\omega \varepsilon
$$

with $\varepsilon$, being the time difference. The plot of the intensity difference $\Delta$ as a function of time is represented in Fig. 5.

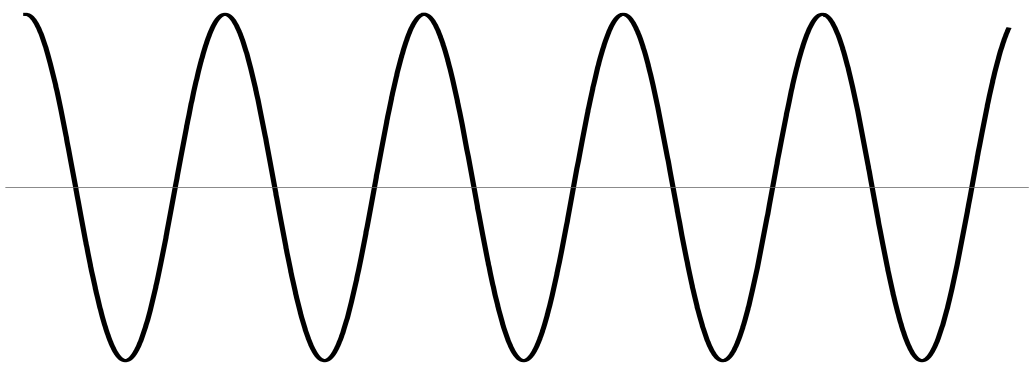

Figure 5. Plot of the intensity difference $\Delta(\varepsilon)$. 
If the two paths are equal the time difference is zero, and the intensity reaches its maximum. By progressively increasing the one-way trip $\ell$ one gets the intensity difference for the one-way travel time as plotted in Fig. 5 .

In these conditions, from the time $\varepsilon=\delta / \omega$ determined experimentally, the one-way velocity of light, $c_{\rightarrow}=\ell / \Delta t$ may be easily determined.

This interferometric process has some advantages over the simple direct detection, since it puts practically no theoretical limits on the relative experimental precision of the measurement. The absolute time error using interferometric procedures, $\Delta t_{0}$, is a fraction of the period of the light. In this case we have

$$
\Delta t_{0}=\alpha \tau=\alpha 10^{-15} s, \quad 0 \leq \alpha \leq 1
$$

Again, assuming that we have $\ell=3 \mathrm{~cm}$, and for $\alpha=.1$, the relative time error would be about $\varrho \sim 10^{-6}$.

An eventual variation of the one-way speed of the light with the relative velocity $v_{i}$ of the experimental platform relative to the medium may also be studied. For that, it is only necessary to make the experiment with different relative velocities.

\section{Conclusions}

It was shown that it is experimentally feasible to study a hypothetical variation on the one-way speed of light when the medium moves relatively to experimental device. In addition, a feasible experimental way for circumvent Poincaré's curse was presented.

Acknowledgements. This work has been funded by FCT (Portugal) through CFCUL (project UID/FIL/00678/2013).

\section{References}

1. Albert A. Michelson, Studies in Optics, Phoenix Books, The University of Chicago Press, Chicago, 1962.

2. Albert A. Michelson and Edward W. Morley, "On the Relative Motion of the Earth and the Luminiferous Ether", American Journal of Science, vol. 34, pp. 333-345, 1887.

3. Albert Einstein, On the electrodynamics of moving bodies, translation from Zur Elektrodynamik bewegter Körper, in Annalen der Physik. vol. 17, p.891, 1905, in The Principle of Relativity, Methuen and Company, Ltd. of London, 1923.

4. F. Selleri, in Frontiers of Fundamental Physics, eds. M. Barone and F. Selleri, Plenum, New York, 1994.

5. F. Selleri, Found. Phys. Vol. 27, p. 1527, 1997.

6. A. Einstein, Les Théories de la Relativité Restreinte et Géneralisée Mise á lá Portée de Tout le Monde, Gauthier-Villars, Paris, 1921.

7. H. Reichenbach, The Philosophy of Space ETime, Dover, New York, 1958.

8. F. Selleri, Chin. Jour. Syst. Eng. El. vol. 6, p. 25, 1995.

9. H. Poincaré, Rev. Metaphysique et Morale vol. 6, p.1, 1898.

10. J.R. Croca, Eurhythmic Physics, or Hyperphysics, The Unification of Physics, Lambert, Berlin, 2015.

11. G. Sagnac, "L'éther lumineux démontré par l'effet du vent relatif d'éther dans un interféromètre en rotation uniforme.", C. R. Acad. Sci., vol. 157, no. 17, pp. 708-710 (1913): 7; Sagnac, "Sur la preuve de la réalité de l'éther lumineux par l'expérience de l'interférographe tournant.", C. R. Acad. Sci. vol. 157, no. 25, pp. 1410-1413, 1913; Sagnac, "Effet tourbillonnaire optique. La circulation de l'éther lumineux dans un interférographe tournant." J. de Phys. vol. 4, pp.177-195, 1914. In the last paper Sagnac developed the entire theory of his experiment, while the Comptes Rendus papers were extracted from this.

12. Ruyong Wang, Y. Zheng, A. Yao and D. Langley, Phys. Lett. A vol. 312, pp. 2003; Ruyong Wang, Yi Zheng, Aiping Yao, Generalized Sagnac Effect, Physical Review Letters vol. 93, p. 143901, 2004. 